\title{
Éditer le manuscrit inachevé
}

Vraies et fausses vertus du numérique

\section{Serge Linkès}

\section{(2) OpenEdition}

\section{Journals}

Édition électronique

URL : http://journals.openedition.org/recherchestravaux/106

DOI : 10.4000/recherchestravaux.106

ISSN : 1969-6434

Éditeur

UGA Éditions/Université Grenoble Alpes

Édition imprimée

Date de publication : 15 juin 2008

Pagination : 185-199

ISBN : 978-2-84310-125-0

ISSN : 0151-1874

Référence électronique

Serge Linkès, « Éditer le manuscrit inachevé », Recherches \& Travaux [En ligne], 72 | 2008, mis en ligne le 15 décembre 2009, consulté le 03 février 2021. URL : http://journals.openedition.org/ recherchestravaux/106; DOI : https://doi.org/10.4000/recherchestravaux.106 


\section{Éditer le manuscrit inachevé : vraies et fausses vertus du numérique ${ }^{r}$}

Que sait-on de la genèse de Lamiel ?

Il y a bien ces quelques mots griffonnés au dos d'une lettre, qui révèlent qu'au printemps i 839 Stendhal décida d'écrire les aventures d'Amiel, une jeune fille entrevue au détour d'une rue. Mais suffisent-ils à rendre compte de ce projet qui l'occupa jusqu'à sa mort?

L'auteur nous a laissé son manuscrit en l'état - il meurt après une séance de dictée chez son copiste favori, le fameux Bonavie ${ }^{2}$ - et les éditions posthumes nous ont fourni les «textes» d'un roman sans fin qui est aujourd'hui l'une des œuvres de Stendhal les plus sujettes à controverse. Les critiques, le plus souvent mus par la curiosité de savoir ce qui se cache derrière le texte, ont saisi l'intérêt que pouvait avoir ce roman sibyllin qui semble vouloir montrer ce qui a toujours été caché aux yeux du lecteur : savoir comment Stendhal écrivait ses romans, savoir comment il élaborait le caractère de ses personnages, savoir... Encore fallait-il que les brouillons informes, cachés jusque-là aux yeux des profanes, se métamorphosent en œuvre consacrée.

L'inachèvement fait que la connaissance que l'on peut avoir de cette œuvre est donc toujours tributaire de l'édition que l'on consulte. Éditer Lamiel comporte donc des risques, il suffit de lire les préfaces des différentes éditions pour s'en convaincre. Chaque éditeur semble condamné à critiquer l'édition précédente et à dresser la liste des erreurs commises dans le déchiffrement du texte.

I. En complément de cet article, un diaporama a été mis en ligne sur le site de la MSHAlpes : http://www.msh-alpes.prd.fr/Actualites/Manuscrits.htm.

2. Sur ce point voir S. Linkès, «Le manuscrit de Lamiel: la fin d'une énigme?», Le dernier Stendhal I $837-1842$, textes réunis par M. Arrous, Saint-Pierre du Mont, Eurédit, 2000. 
Peut-il en être autrement? Lamiel est avant tout un brouillon. Stendhal l'a abandonné un soir de mars I 842, en oubliant de signaler ce qu'il voulait en faire. Comme le précise Jacques Laurent dans une préface de ce roman : «Le Lamiel qu'il nous a laissé n'est donc pas seulement une œuvre inachevée, c'est une masse qui n'a pas encore sa forme... ${ }^{3}$ » Il n'y a donc pas que l'inachèvement qui pose problème dans le dossier du manuscrit de Lamiel. L'organisation des volumes ${ }^{4}$, la diversité des écritures et des supports, la datation des différents ensembles, des fragments, l'enchaînement des versions... sont autant d'éléments qui font de ce manuscrit une énigme.

Pourtant, la première lecture de ce dossier complexe laisse pressentir qu'il détient peut-être quelques clés pour comprendre les procédés de la composition stendhalienne. Car, contrairement à certains de ses contemporains, Stendhal se désintéressait systématiquement des manuscrits une fois les œuvres publiées. Ses procédés de composition restent donc mal connus, et l'on est réduit seulement à supputer chez lui ce que chez d'autres on peut vérifier. Mais il y a Lamiel, et par son inachèvement même, cette œuvre est une source inépuisable d'informations sur les processus de la création romanesque de Stendhal. L'examen d'un dossier de genèse ne peut sans doute expliquer à lui seul l'« Art de composer les romans'» comme l'appelait Stendhal, mais il fournit certains éléments d'information susceptibles de nous aider à progresser dans la compréhension des œuvres éditées de son vivant. Toutefois, avant de pouvoir s'interroger sur la composition de ce roman et prétendre en tirer quelques leçons, il convient de s'interroger sur la forme éditoriale la plus adaptée à la complexité de ce dossier. L'édition numérique, par son adaptabilité et la relative liberté qu'elle donne à l'éditeur, semble indiquée dans ce cas. Malgré certaines limites, elle permet de prendre en compte l'objet dans son ensemble, à travers sa dimension intellectuelle et sa dimension matérielle, afin de proposer au lecteur un objet virtuel mais structuré, lisible et adapté à l'interprétation critique.

\section{Lire Lamiel: le manuscrit et la tradition éditoriale}

Si les éditeurs qui ont publié le texte de Stendhal se sont attachés à décrypter scrupuleusement l'écriture de l'auteur, aucun ne s'est véritablement interrogé jusqu'ici sur les problèmes que pose le manuscrit en lui-même. Pour que Lamiel

3. J. Laurent, «Préface de Lamiel», Stendhal, Le Ronge et le noir, La Chartreuse de Parme, Lamiel, Armance, Robert Laffont, I980, p. I 3.

4. Le manuscrit de Lamiel est conservé à la Bibliothèque municipale de Grenoble et comporte 5 volumes (R 297 I et II, R 298 I et II, N 3457) soit 7I 8 feuillets.

5. R $297 \mathrm{I}$, folios 2 à 4 . 
livre ses secrets au lecteur et puisse le renseigner utilement sur «l'écriture stendhalienne», il importe de se pencher d'abord sur l'ensemble que constitue ce dossier manuscrit et sur la méthode employée. Car un manuscrit contient bien plus que ce qui est inscrit sur ses pages, et la critique génétique a développé une méthodologie et des outils théoriques qui permettent de mettre en évidence ce contenu et d'en tirer de précieux renseignements. À partir d'un tel examen, nous avons tenté d'enrichir cette méthodologie en nous interrogeant sur la méthode à employer pour l'analyse d'un manuscrit inachevé et en nous appuyant sur les nouvelles technologies de l'information ${ }^{6}$. Cette nouvelle approche du manuscrit stendhalien nous a permis d'aborder un des rares domaines encore inexplorés des études stendhaliennes. Ainsi, en nous interrogeant sur le rôle des copistes et en procédant à une analyse matérielle du manuscrit de Lamiel, avons- nous dévoilé des aspects inédits de la dernière œuvre de Stendhal ${ }^{8}$ que seule une édition génétique complète révèlera au lecteur9. En effet, le principal intérêt de l'analyse génétique est de permettre d'établir, dans le cas de l'inachevé en particulier ${ }^{10}$, un avant-texte qui tienne compte de tous les aspects intellectuels et matériels du manuscrit, sur lequel peut se fonder le travail éditorial et finalement le discours critique. Dans le cas de Lamiel, cet examen était d'autant plus important que son absence dans toutes les éditions antérieures rendait plus incertaine une analyse critique du roman. L'autre enjeu de cet examen était de mettre à jour les versions successives afin de mieux cerner le projet initial de Stendhal, mais aussi son évolution au fil du temps.

L'inachèvement est donc au centre des difficultés rencontrées dans l'examen de ce manuscrit, et la question peut se poser dans ces simples termes : existe-t-il un texte de Lamiel? Dans le domaine de la critique génétique, le texte «coïncide avec la publication imprimée qui fait passer l'écrit d'un statut autographique et privé à un statut allographique et public ${ }^{11} \gg$. De ce point de

6. Voir $\mathrm{Cl}$. Bustarret et $\mathrm{S}$. Linkès, «De Muse en Argolide, ou la codicologie à l'ère du numérique», dans le présent volume, p. i i 9.

7. Toutefois nous n'oublions pas les travaux pionniers de G. Rannaud et de S. Sérodes.

8. S. Linkès, "Genèse de Lamiel, le chaînon manquant», thèse de Doctorat sous la direction de B. Didier, Université Paris VIII, 2000.

9. Pour l'édition critique : Stendhal, Lamiel, Romans III, texte établi et annoté par S. Linkès, Gallimard, «Bibliothèque de la Pléiade», à paraître en 2008. Pour l'édition génétique : Stendhal, Lamiel, texte établi et annoté par S. Linkès, Champion «Textes de littérature moderne et contemporaine», à paraître en 2008 (éditions diplomatique papier et électronique).

Io. Sur les problèmes de l'inachèvement voir J.-L. Lebrave, «L'écriture inachevée» in D. Budor et D. Ferraris, Objets inachevés de l'écriture, Presses de la Sorbonne nouvelle, 200 I, p. I 930. On corrigera toutefois le classement de Lamiel parmi les œuvres «abandonnées» de Stendhal, puisque, comme on le sait maintenant, l'inachèvement de cette œuvre est totalement involontaire et seulement dû au décès au décès de l'auteur.

I I. P.-M. de Biasi, La Génétique des textes, Nathan, 2000 p. 29. 
vue, le texte de Lamiel existe, mais n'existe pas. Il n'existe pas, car on le sait, Stendhal n'a jamais publié une œuvre intitulée Lamiel de son vivant; mais il existe, comme le prouve la facilité de se procurer un livre portant le titre de Lamiel écrit par Stendhal. Ce paradoxe est d'autant plus important qu'il conditionne à la fois la vision littéraire et critique de l'œuvre, mais également l'examen du manuscrit. Encore une fois, c'est l'inachèvement qui nous met dans cette situation paradoxale où le texte édité se confond plus ou moins avec son dossier de genèse. En effet, le manuscrit de Lamiel est le passage obligé de chaque éditeur, qui marque plus ou moins celui-ci, et parfois même physiquement $^{12}$. Depuis I 889 , ce phénomène a fait naître une sorte de tradition éditoriale qui fait que Lamiel ne sort jamais indemne d'une nouvelle publication. Parallèlement à cette tradition éditoriale s'est développé un discours critique. Ce discours a évolué en fonction des différents principes adoptés par les éditions qui se sont succédé. Mais c'est certainement l'édition de Victor Del Litto au Cercle du Bibliophile ${ }^{13}$ qui a inauguré la nouvelle voie d'un discours critique s'interrogeant sur les mystères de la composition stendhalienne à partir de ce roman. En effet, postulant l'existence de deux textes distincts, la critique a entériné l'existence des textes élaborés par cette édition. Ainsi est apparue une nouvelle théorie du texte de Lamiel $^{14}$ qui a souvent conditionné l'idée que l'on se fait du manuscrit lui-même. Relayé par la critique qui a institutionnalisé

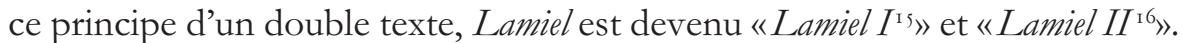
Cette idée, développée par F. W. John Hemmings (art. cit., p. 292-293), a été largement diffusée au sein de la critique stendhalienne au point que l'on a vu s'ajouter un «Lamiel IIII7》 pour concrétiser les derniers «ajouts» et remaniements datés de i84I et i 842. Le problème posé par l'édition de Victor Del Litto réside dans le fait que cette production a été confondue avec le texte, voire avec le manuscrit lui-même. Gérard Genette avait pourtant brillamment résumé le paradoxe de l'œuvre inachevée chez Stendhal : «Le texte stendhalien, marges et bretelles comprises, est un. Rien ne permet d'y isoler cette sorte de super-texte précisément élaboré qui serait, ne varietur, l'œuvre de Stendhal. Tout ce que trace la plume de Beyle (ou sa canne, ou son canif, ou

I 2. Voir sur ce point S. Linkès, «Genèse de Lamiel, le chaînon manquant», op. cit., partie I, chapitre II, V: «La constitution du manuscrit de Lamiel».

I 3. Stendhal, Lamiel, texte établi, annoté et préfacé par V. Del Litto, Genève, Cercle du Bibliophile, I97 I.

I 4. Voir principalement F. W. John Hemmings, «À propos de la nouvelle édition de Lamiel. Les deux Lamiel. Nouveaux aperçus sur les procédés de composition de Stendhal romancier», Stendhal Club, no 6o, 1973.

I 5 . La version du roman que l'on supposait écrite entre le $\mathrm{I}^{\text {er }}$ octobre et le 3 décembre 1839. 16. La version du roman que l'on supposait écrite entre le 3 janvier et le 23 novembre i 840. I 7. Chr. W. Thompson, Lamiel fille du feu, Essai sur Stendhal et l'énergie, L'Harmattan, I 997. 
Dieu sait quoi) est Stendhal, sans distinction ni hiérarchie ${ }^{18} . »$ Finalement, cette édition ne donne pas un texte de Lamiel, mais un avant-texte inexact. Cet adjectif dépréciatif n'entame en rien la valeur de ce travail éditorial, il est la conséquence d'un manque d'outils théoriques, qui faisaient encore défaut à cette époque. Comme le précise Pierre-Marc de Biasi, l'avant-texte est une «entité qui n'existe pas en dehors du geste scientifique qui le constitue, mais il ne s'agit pas encore à proprement parler d'une interprétation de la genèse : on ne classe pas et on ne déchiffre pas les documents en fonction de présupposés interprétatifs» (op. cit., p. 3I). Cette mise en garde ne s'applique pas simplement aux généticiens du texte, mais à tous ceux qui, de près ou de loin, se confrontent à l'univers du manuscrit. Il est évident que l'édition d'un roman inachevé comme Lamiel nécessite une étude approfondie du dossier (qui ne peut faire abstraction de sa double dimension matérielle et intellectuelle) dont l'édition même doit être le reflet.

La consultation simultanée du manuscrit de Lamiel et de l'édition de Victor Del Litto révèle l'absence de toute analyse matérielle et une démarche téléologique fondée sur un texte idéal de Lamiel qui sont à l'origine des failles de cet avant-texte. On peut noter aussi la présence de deux présupposés interprétatifs : d'abord celui d'une chronologie de la rédaction de l'œuvre qui n'a jamais été remise en cause par cet éditeur et qu'il s'est efforcé d'appliquer coûte que coûte au classement des brouillons; ensuite celui du principe, peu approprié à l'écriture stendhalienne, que l'autographe précède forcément le manuscrit du copiste. Ce dernier point semble avoir guidé les éditeurs qui ont succédé à Victor Del Litto pour les éditions de poche au point que le véritable premier jet ${ }^{19}$, dicté par Stendhal à son copiste Bonavie au mois de mai 1839 (soit plus de 70 folios ${ }^{2 \circ}$ ), est devenu la seconde version du texte, simplement parce qu'il est plus conforme, dans l'idée que le critique se fait de la création littéraire, que l'autographe précède la copie. On peut aussi s'interroger sur le fait que les critiques n'aient pas considéré ces éditions comme ce qu'elles doivent être : des avant-textes qui ne sont que des projections critiques de l'éditeur ${ }^{21}$ sur les brouillons de Lamiel. L'édition de Victor Del Litto, par sa qualité, sa forme et ses principes fort justes, semble être devenue non seulement l'avant-texte

I 8. G. Genette, Figures II, Seuil, I969, p. I69.

I 9. Sur ce point, voir S. Linkès, «Le manuscrit de Lamiel: la fin d'une énigme?», art. cit.

20. Voir «Le cas Lamiel», Cl. Bustarret et S. Linkès, «De MUSE en ARGOLIDE, ou la codicologie à l'ère du numérique», dans le présent volume, p. I i 9 et p. I 85.

2I. V. Del Litto précisait pourtant clairement: «Le travail de mise au point auquel je me suis livré n'exclut ni les amendements ni les améliorations.» (Préface de l'édition de Lamiel, op. cit., p. XIX). Cette mise en garde ne peut toutefois prendre toute sa valeur sans une connaissance minimale de l'objet matériel qu'est le manuscrit de Lamiel. En l'absence de cette référence, il est difficile de se rendre compte de la difficulté d'une telle entreprise. 
d'un texte qui n'existe pas, ou qui n'existe qu'à travers son édition, mais aussi le dossier génétique de l'œuvre. Ainsi, la confiance des critiques à l'égard de ce Lamiel «intégral et authentique ${ }^{22} »$ fait qu'on s'est fié plus facilement à l'édition qu'au manuscrit lui-même.

Tout éditer, c'est la volonté qui anima les principaux éditeurs de Lamiel. Mais il faut constater que, dans le cas de l'inachevé, celle-ci prouve ses limites même si elle a le mérite de présenter un texte de Lamiel plus proche du manuscrit. Si les principes choisis par les éditeurs sont dans l'ensemble justes pour une édition critique, ils sont soumis à une connaissance extrêmement précise du dossier. Ainsi, on peut dire que c'est à cause de la justesse de son premier principe que l'édition de Victor Del Litto pose problème : il est en effet regrettable de se tromper dans la chronologie de la rédaction d'une œuvre, lorsqu'on suit celle-ci pour établir le texte.

Cependant, il faut également reconnaître que la forme de l'édition critique n'est guère adaptée au cas de l'inachevé. Le manuscrit est vite à «l'étroit» dans cette forme d'édition. Des problèmes se posent, non seulement pour les versions des textes qui ne sont pas assez élaborées pour être éditées telles quelles, mais également pour les divers fragments et notes qui parsèment les brouillons, qui ne semblent jamais être à leur place dans ce type d'édition. C'est peut-être simplement que le manuscrit ne peut être édité convenablement sur un support papier. Ce paradoxe résume toute la difficulté de l'édition scientifique de l'inachevé. Comment mettre à plat un objet que l'on ne peut comprendre qu'à travers toutes ses dimensions, son relief, ses correspondances et son réseau intratextuel? Dans le manuscrit de Lamiel tout est lié sans l'être véritablement, tout est proche intellectuellement mais éloigné matériellement, et aucun papier n'est pour le moment capable de rendre de tels effets. C'est pourquoi on pourrait penser que la forme idéale de l'édition scientifique d'une œuvre inachevée serait plus proche d'une édition génétique que d'une édition traditionnelle.

\section{Re-lire Lamiel, édition génétique et édition numérique}

Si l'édition génétique semble plus appropriée pour présenter le dernier roman de Stendhal, c'est parce que son but est plus de communiquer au lecteur les phases invisibles de l'élaboration d'une œuvre que l'œuvre elle-même. Nous nous sommes efforcé de montrer qu'il n'existait pas de texte de Lamiel, mais plutôt un ensemble de projets que Stendhal a réunis sous ce titre; l'édition 
génétique, qui «n'établit pas un texte, mais cherche à rendre lisible et intelligible une étape de sa genès ${ }^{23}$ », est donc particulièrement indiquée pour présenter ce laboratoire de l'invention stendhalienne qu'est Lamiel.

L'édition génétique peut s'envisager sous deux formes : l'édition verticale, qui vise l'ensemble de la genèse et qui reconstitue la totalité du processus d'écriture, et l'édition horizontale, qui transmet un moment particulier de ce processus. Qu'elle soit verticale ou horizontale, cette édition peut se présenter sous des aspects assez différents. Il faut bien avouer qu'aucune règle ne régit véritablement l'édition génétique, qui n'en est qu'à ses débuts, mais une harmonisation des principes éditoriaux s'élabore peu à peu ${ }^{24}$.

Car l'enjeu de l'édition génétique est clair : il s'agit de «représenter dans la "bidimensionalité" des pages un processus génétique dont on s'est pourtant appliqué à montrer que sa propriété est d'ajouter à l'écrit, qui est bidimensionnel, une troisième dimension, qui est celle du temps [...]. On s'étonne que ce nouvel ensemble ne veuille plus réellement rentrer dans le cadre de l'espace-page» (ibid.). Le chercheur doit donc tenter de dépasser cet espace restreint par le biais de la transcription. La transcription «linéarisée» est certainement la plus pratiquée par les éditeurs. Cette méthode, qui est plus proche de l'édition traditionnelle, fait apparaître les ratures et les ajouts grâce à un code de transcription parfois aussi complexe que les opérations scripturales de l'auteur elles-mêmes. L'autre méthode, qui consiste à reproduire la disposition topographique de l'original en respectant les blancs, les renvois, les marges..., plus proche de la réalité du manuscrit, dite «transcription diplomatique», permet de découvrir les habitudes de mise en page de l'auteur, souvent très révélatrices de son mode opératoire.

Toutefois, même dans le cas de l'édition diplomatique, le problème de la multidimensionnalité du manuscrit reste entier. L'édition diplomatique peut effectivement rendre sur une même page les versions successives d'un texte à travers un réseau de signes conventionnels établissant un code de transcription, ou dans une reproduction fac-similé de l'original. Mais il faut dans ce cas établir un appareil critique extrêmement précis qui permette aux lecteurs de retrouver les réseaux de communication existant dans le manuscrit. Il sera très difficile de signaler tous les renvois, toutes les interventions de l'auteur sur le brouillon sans réduire de façon considérable la lisibilité du texte. De

23. P.-M. de Biasi, op. cit., p. 69.

24. Il y a quelque temps, Almuth Grésillon constatait que "dans cette "Babel génétique" [...] chacun prétend que la genèse de "son" auteur est à nulle autre pareille et mérite donc un système de représentation et de codage bien à elle» (A. Grésillon, Eléments de critique génétique, PUF, I 974, P. I 2 I) 
même, quelle que soit la méthode de transcription adoptée, la dimension temporelle du manuscrit reste difficile à rendre.

Il faut donc constater que malgré toutes les qualités de l'édition génétique, le manuscrit, qui se construit à travers un réseau de correspondances, est impossible à reproduire sous la forme traditionnelle du livre. Ce réseau peut être rapproché de l'hypertexte ${ }^{25}$, qui est le principe de base de l'élaboration des sites Internet ${ }^{26}$. Toutefois, les réseaux observés dans les manuscrits d'auteurs intègrent une dimension temporelle et sont beaucoup plus aléatoires et complexes; ainsi, il ne suffit pas d'appliquer les techniques développées pour Internet aux pages de Lamiel pour obtenir une édition numérique.

Le terme «hypertexte», inventé par Ted Nelson en $1965^{27}$, est longtemps resté confiné aux cercles des chercheurs avant de connaître un succès croissant. Ce mot désignait dans l'esprit de Ted Nelson un dispositif de lecture et d'écriture informatique, connu sous le nom de PROJECT XANADU ${ }^{28}$, renonçant à la linéarité du document pour y préférer des principes d'analogies. Ce terme s'est vite doté d'une polysémie à laquelle la critique littéraire a contribué sans forcément s'en rendre compte ${ }^{29}$. Les travaux de George P. Landow ${ }^{30}$ révèlent effectivement les analogies entre la pensée des théoriciens du texte comme Barthes, Derrida, Bakhtine et Genette et de l'hypertexte informatique (op. cit., p. I-6, et 53-68). Les théories structuralistes et poststructuralistes définissant la pensée comme un réseau induisent les notions de structures, de relations entre des unités ou des fragments (les célèbres lexies de Roland Barthes ${ }^{31}$ ). On retrouve naturellement ces relations de transtextualité dans l'hypertexte informatique, mais l'on doit tempérer les propos de George P. Landow en

25. Dans sa définition minimale, l'hypertexte est un système constitué par un ensemble de textes et par des liens qui les unissent, permettant au lecteur de passer de l'un à l'autre selon ses besoins. C'est sur ce principe que sont constitués les pages et les sites Internet.

26. Pour un historique de la notion $d^{\prime}$ «hypertexte» et une analyse épistémologique du fonctionnement hypertextuel, voir J. Clément, «Du texte à l'hypertexte : vers une épistémologie de la discursivité hypertextuelle», Hypertextes et hypermédias : réalisations, outils, méthodes, J.-P. Balpe, A. Lelu, I. Saleh (coordinateurs), Hermès, I 995 ; article consultable à l'adresse : http://hypermedia.univ-paris8.fr/jean/articles/discursivite.htm.

27. Il faut toutefois préciser que le concept lui-même est à attribuer à V. Bush. Bien avant l'invention des technologies de l'information, V. Bush proposa, dans son article «As We May Think», Atlantic Monthly, juillet I 945, une machine (MEMEX - acronyme pour memory extender) capable de créer automatiquement des liens entre différents supports, de naviguer à travers la toile des informations.

28. The Original Hypertext Project: http://xanadu.com/.

29. Ce phénomène est évidemment accentué par le fait que l'hypertexte a été détourné par des écrivains pour produire un nouveau genre littéraire : l'hypertexte fictionnel.

30. G. P. Landow, Hypertexte 3.0, Critical Theory and New Media in an Era of Globalisation, Baltimore, The Johns Hopkins University Press, 2006.

3I. R. Barthes, $S / Z$, Seuil, «Points», I 970. 
précisant la nature même de la transtextualité chez les uns et les autres : pour les théoriciens du texte, la transtextualité est un phénomène observable et observé; pour les théoriciens de l'hypertexte informatique, il s'agit d'un principe fondateur qui modèle la création du document. Cette convergence ${ }^{22}$ s'applique donc plus facilement à l'hypertexte fictionnel ${ }^{33}$ (créé pour ce type de support et qui se situe à la convergence même de ces deux formes de transtextualité) qu'au texte «traditionnel», même si leur édition hypertextuelle permet souvent de souligner le phénomène d'intratextualité. C'est dans le domaine de l'intertextualité que les théories de l'hypertexte informatique et du texte littéraire se recoupent le plus. Par exemple, le projet XANADU de Ted Nelson suppose non seulement l'existence de relations entre les documents, mais aussi le repérage des liens intertextuels et bidirectionnels entre les œuvres ${ }^{34}$. Mais on trouve une convergence encore plus troublante dans la description que fait Gérard Genette du phénomène hypertextuel littéraire ${ }^{35}$. En s'interrogeant sur une littérature qui s'inscrirait dans la tradition, il définit, par le terme «hypertexte», le «texte dérivé d'un texte antérieur par transformation $\aleph^{36}$.

C'est finalement sur les différentes acceptions du terme «hypertexte» que nous nous pouvons nous appuyer pour élaborer une édition numérique et génétique du manuscrit inachevé de Stendhal. Les travaux des généticiens du texte ont montré que l'imprimé et ses caractéristiques (l'unicité, la linéarité et l'achèvement de l'œuvre) n'étaient que la partie émergée d'un processus intellectuel de création plus complexe qui peut s'apparenter à l'hypertexte. Jean-

32. G. P. Landow, «Cause or Convergence, Influence or Confluence?», op. cit., p. 65.

33. Sur l'hypertexte de fiction, voir J. Clément, «Hypertextes et mondes fictionnels ou l'avenir de la narration dans le cyberespace», Les Enjeux du virtuel, N. Nel (directeur), L'Harmattan, $200 \mathrm{I}$.

34. Voici comment T. Nelson résume son projet : «On interprète souvent à tort Xanadu, le projet original de l'hypertexte, comme une tentative pour créer le World Wide Web. Mais $X a n a d u$ a toujours eu une ambition beaucoup plus vaste : proposer une forme globale et complète de littérature; où les liens ne se brisent pas à chaque changement de version; où les documents peuvent être mis côte à côte, comparés et annotés de près; où il est possible de connaître le contexte d'origine de chaque citation; et qui intègre un dispositif de droit d'auteur - une convention littéraire, juridique et commerciale - autorisant, sans conflit ni négociation, la pratique de citation, sans limite de temps ou de quantité. [...] La littérature électronique sérieuse (à des fins de connaissance, de travail en coopération ou de débat approfondi) doit permettre des liens bidirectionnels, à profusion; et une réutilisation systématisée, s'appuyant sur une recherche facile à travers les versions et citations.» (Th. Nelson, extrait de «Transcopyright : Pre-Permission for Virtual Republishing», Educom Review (janvier/février 1997), traduction Ministère de la Culture, 200I, http://www.culture.gouv.fr/culture/actualites/conferen/tasca200I/extraits-nelson.htm).

35. La réflexion menée par G. P. Landow sur les théories de G. Genette se limite à celle exposée par le critique dans la traduction anglaise de Figures I. On ne trouve donc pas de réflexion sur l'emploi du terme «hypertexte» qui n’apparaît que dans Palimpsestes en i 982.

36. G. Genette, Palimpsestes, Seuil, «Poétique», I 982 , p. I6. 
Louis Lebrave, notamment, a reconnu une pratique anticipant l'hypertexte chez Stendhal : «L'écriture est ici bien plus qu'un simple support de stockage jouant le rôle d'extension externe de la mémoire, elle est à la fois trace sur un support et processus produisant cette trace ${ }^{37}$.» En effet, Stendhal avait même pris l'habitude de dépasser les frontières de l'imprimé en déstructurant le livre; pour cela il n'hésitait pas à faire dérelier les œuvres pour y ajouter des pages blanches afin d'annoter les documents au fil de la lecture ${ }^{8}$... Mais le brouillon stendhalien lui-même se rapproche déjà fortement de cette pratique hypertextuelle : les feuillets réunis dans des dossiers le plus souvent identifiés par leur couleur (rouge et noir, ou vert et bleu comme chez Lamiel) restent mobiles tant qu'ils ne sont pas reliés ${ }^{39}$, et le système de paginations élaboré par Stendhal lui permet de garder la trace des déplacements de chaque feuillet au fur et à mesure de l'élaboration des différentes versions. De même, l'auteur a élaboré un système complexe de renvois pour dépasser le cadre de la page, lui permettant de garder les traces des différentes campagnes de corrections, de dictées ou d'écriture.

Le manuscrit stendhalien défie donc la linéarité pour se lire sur l'axe paradigmatique. Si cette pratique contribue à ce que Gérard Genette appelle $l^{\prime}$ «aporie du stendhalisme ${ }^{40} »$, elle illustre également l'aspect hypertextuel de la relation génétique qui

se ramène constamment à une pratique d'autotransformation, par amplification, par réduction ou par substitution. Si inépuisable que soit son champ d'étude et si complexes que soient ses opérations, elle est bien un cas particulier [...] de l'hypertextualité telle que définie ici : tout état rédactionnel fonctionne comme un hypertexte par rapport au précédent, et comme un hypotexte par rapport au suivant. De la première esquisse à la dernière correction, la genèse d'un texte est une affaire d'auto-hypertextualité ${ }^{4 \mathrm{I}}$.

Si l'hypertextualité peut se reconnaittre dans la construction de l'avanttexte, elle peut également être envisagée comme un mode de lecture de celuici. Dans le cas de l'inachevé, c'est même la seule lecture que l'on puisse en faire. En effet, comme on peut le voir dans le dossier Lamiel (fig. I), le premier jet du mois de mai I 839 peut être considéré comme l'hypotexte des versions suivantes.

37. J.-L. Lebrave, «Hypertextes - Mémoires - Écriture», Genesis nº 5, I994.

38. Anticipant ainsi certaines revendications du Web 3.0.

39. Mais, comme nous l'avons vu, aucune reliure n'est vraiment définitive chez Stendhal.

40. «Un texte fragmenté, morcelé, lacunaire, répétitif, et par ailleurs infini, ou pour le moins indéfini, mais dont aucune partie ne peut être séparée de l'ensemble. Qui tire un seul fil doit emporter toute la nappe, avec ses trous, et jusqu'à son absence de bords» (G. Genette, Figures II, op. cit., p. 176).

4I. G. Genette, Palimpsestes, op. cit., p. 55 I. 


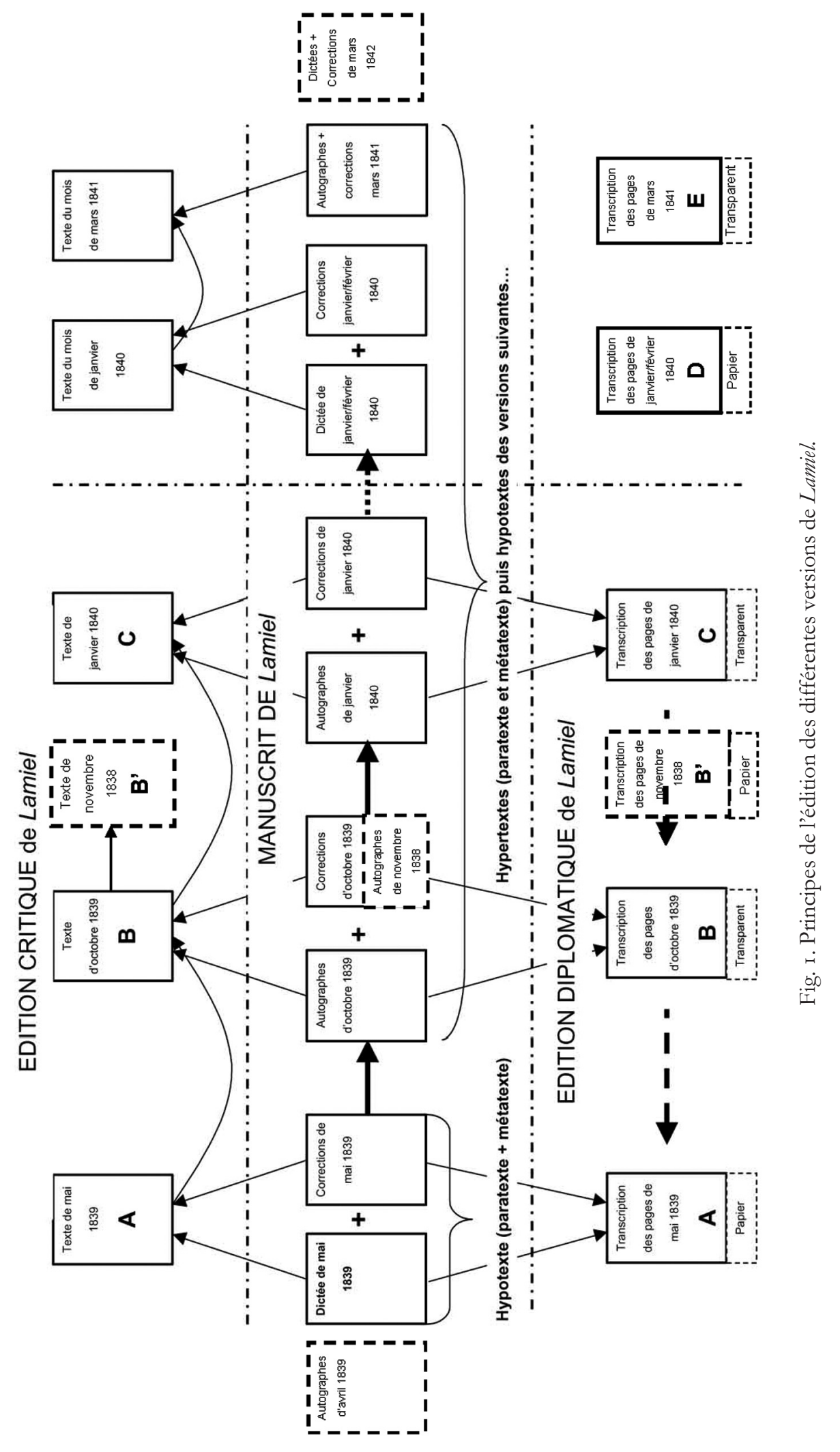


L'hypertexte du mois d'octobre i 839 obtenu par autotransformation - se limitant dans ce cas à des ajouts et des corrections - devient lui-même l'hypotexte de l'hypertexte du mois de janvier i 840. Cependant, le dernier maillon de cette chaîne d'autotransformation n'est pas pour autant l'hypertexte de référence, mais un moment arrêté de l'élaboration d'un texte. Il ne peut en aucun cas remplacer la version finale qui aurait été éditée du vivant de l'auteur. Dans le cas de Lamiel, on sait que les transformations du texte se sont poursuivies jusqu'à la mort de l'auteur, et rien ne permet d'affirmer qu'une version plutôt qu'une autre puisse être l'aboutissement du texte. De même, il est évident que ce roman inachevé est illisible sans le paratexte et le métatexte qui s'inscrivent eux-mêmes dans la chaîne hypertextuelle.

Car l'inachèvement de l'œuvre pose toujours le même problème. L'hypertexte qui se construit sur une relation entre un texte «source» et un texte «but» s'inverse dans le cas de l'inachevé. Cependant, les combinaisons sont multiples puisque les liens s'établissent non seulement entre les pages, mais également entre les différents éléments (et entre autres les «lexies», pour reprendre le terme de Roland Barthes) qu'elles contiennent. Ainsi, l'hypertexte génétique, s’inspirant des raisonnements et des concepts élaborés par la linguistique, cherche à croiser l'axe syntagmatique du flux de l'écriture et l'axe paradigmatique des réécritures. Il est toutefois délicat de construire les paradigmes des variantes quand la déclinaison de la lexie variante est incomplète. Le paradigme devra donc se développer à partir de la première version de cette lexie, pour permettre au lecteur de suivre toutes les variantes déclinées par l'auteur, tout en sachant qu'il n'existe pas de lexie idéale, c'est-à-dire entérinée par l'édition.

L'édition diplomatique et génétique du premier jet de Lamiel que nous avions proposée en annexe de notre thèse (op. cit.) coupait net les relations hypertextuelles qui s'établissent dans le dossier de l'inachevé, puisqu'il s'agissait d'une édition horizontale. Cependant, comme elle est située au début de la genèse, cette partie du dossier Lamiel qui couvre la période du mois de mai I 839 au mois de janvier I 840 peut se comprendre sans présenter l'intégralité du dossier ${ }^{42}$. La figure I permet de synthétiser les principes de cette édition et de l'édition des trois textes obtenus en tenant compte des différentes campagnes de corrections. Le premier jet était logiquement considéré comme l'hypotexte originel, que nous appelions «A». Celui-ci donne naissance en octobre $\mathrm{I} 839$ à un hypertexte que nous appelions «B», qui devient l'hypotexte de l'hypertexte «C». Ce dernier, élaboré en janvier I 840, devenait lui-même

42. Cependant, malgré une relative autonomie, les liens que cette première partie entretient avec l'ensemble du dossier restent évidents. 
l'hypotexte de la version dictée aux copistes romains en janvier i 840. Les éditions en préparation ${ }^{43}$ rétabliront les liens qui unissent ces textes à ceux qui les ont suivis, afin de rendre la totalité de l'enchevêtrement génétique.

Si la critique génétique s'est proposée de renouveler la lecture des œuvres en déplaçant «l'interrogation critique de l'auteur vers l'écrivain, de l'écrit vers l'écriture, de la structure vers le processus, de l'œuvre vers sa genèse ${ }^{44}$ », l'intérêt de l'édition numérique des manuscrits serait finalement de rendre visible, ou plutôt accessible, l'ensemble de ces processus. Les principales étapes de l'analyse génétique (analyse matérielle, délimitation du corpus, repérage des principales phases du travail ${ }^{45}$, classement des différentes versions et des esquisses...) peuvent facilement s'intégrer à l'édition numérique qui s'affranchit des contraintes de linéarité et d'absence de dynamique de l'édition papier.

Par exemple, l'édition numérique a la capacité de donner accès à l'ensemble du corpus constituant le dossier de genèse, là où l'édition papier devait se contenter de renvois bibliographiques. On peut ainsi rassembler dans un même «ouvrage» l'ensemble des pièces manuscrites endogènes de l'œuvre. Dans le cas de Lamiel, bien que le corpus semblât, dans un premier temps, se limiter aux quatre volumes signalés dans les catalogues de la bibliothèque ${ }^{46}$, il fallait l'enrichir de nombreux folios disséminés dans les autres volumes. Ainsi, des ensembles exogènes comme le «Cours de littérature ${ }^{47}$ », les esquisses de Letellier - le principal projet dramatique de Stendhal - et ses diverses réflexions sur la comédie sont venus enrichir les documents de la phase pré-rédactionnelle du dossier. Ces documents autographes, parfois très antérieurs à la rédaction de Lamiel, sont essentiels dans l'élaboration du projet de ce roman, car c'est le même projet qui refait surface ${ }^{4}$. Avec ces éléments rassemblés au sein d'une même édition, Lamiel prend une autre dimension et apparait comme le fruit d'une longue réflexion sur le comique et la comédie.

Ainsi, l'univers multimédia semble particulièrement adapté à l'édition du manuscrit. On peut être tenté alors de dépasser les frontières du dossier de genèse et d'étendre les ramifications de l'œuvre jusque dans son intertextualité la plus poussée. On imagine ainsi la valeur ajoutée d’une édition lorsque celleci peut s'agrémenter des œuvres qui ont contribué à sa genèse : un Rouge et Noir

\footnotetext{
43. Voir note 8.

44. P.-M. de Biasi, op. cit., p. 9.

45. Qui dans le cas du manuscrit inachevé se limite le plus souvent à la phase pré-rédactionnelle (les plans et rédactions antérieurs non aboutis, les notes et documentations diverses...), ainsi qu'à la phase rédactionnelle (structuration, documentation, textualisation...).

46. R 297 I et II, R 298 I et II.

47. «Cours de littérature» (I 840) : R 5896, XV, folios 50- 56.

48. Voir S. Linkès, «De Letellier à Lamiel, la comédie continue», L'Année stendhalienne, nº I, p. $255-280$.
} 
renvoyant aux articles de la Gazette des tribunaux de décembre i 827, un Armance pointant vers Olivier ou le secret, Olivier (Brusson) et finalement (mais pourquoi s'arrêter là...) Das Fräulein von Scudery. Quant au manuscrit inachevé, Lamiel pourrait décliner sa lente gestation des premières tentatives théâtrales de Stendhal jusqu'aux derniers traits de la plume de Bonavie sans oublier ce qu'il doit au Génie du Christianisme, à Madame de Staël, Regnard, Molière, L'Arioste ${ }^{49}$... L'édition numérique peut d'ores et déjà dépasser les contraintes de l'hypertexte arborescent et préférer une forme plus libres ${ }^{\text {so }}$, une structure en étoile dont l'œuvre inachevée serait le point névralgique associant le combinatoire (permettant ainsi d'en explorer les multiples pistes), l'hypermédia et le Web sémantique ${ }^{\text {sI }}$ (afin de relier sémantiquement et d'associer avec pertinence des éléments hétérogènes : images du manuscrit, musique, reproduction d'œuvres picturales)... mais y aura-t-il encore un centre dans cette galaxie éditoriale?

On nous a prévenus que «nul ne peut encore, et sans doute nul ne pourra jamais tracer les limites du corpus stendhalien ${ }^{2} »$, mais la tentation du tout numérique est fortes et l'on imagine déjà le lecteur errant dans une "galaxie de signifiants ${ }^{54} \gg$ et définitivement perdu dans ce XANADU stendhalien. Il revient à l'éditeur de corriger cette tendance à l'explosion de l'information, soit en s'imposant des limites, soit en donnant au lecteur les moyens de contrôler cette profusion. En effet, les possibilités éditoriales qui sont démultipliées par

49. Voir sur ce point S. Linkès, «Les poétiques de Beyle et de Stendhal : rupture ou continuité?», Actes du colloque international «Henri Beyle, un écrivain méconnu», Saint-Pierre du Mont, Eurédit, 2006.

50. Voir J. Clément, art. cit. supra, note 26, et J.-P. Balpe, A. Lelu, I. Saleh, op. cit.

5 I. Voir sur ce point Bulletin de l'Association française pour l'intelligence artificielle, «Dossier Web sémantique» coordonné par Ch. Reynaud, J. Charlet, Ph. Laublet, n 54, avril 2003, p. I6-54, http://afia-france.org/.

52. G. Genette, Figures II, op. cit., p. 175 .

53. Il ne manque finalement qu'une chose pour que l'édition numérique puisse enfin faire oublier le papier : être capable de donner autant de plaisir à la lecture qu'en est capable un simple livre. Ce contact avec l'objet-livre, le tenir en main, en faire défiler les pages sans que le regard se trouble, en prendre grand soin, le sentir lorsqu'il est trouvé chez un bouquiniste... Le plus grand ennemi de l'édition numérique c'est l'absence de matérialisation de ce moment, c'est son interface qui malgré son dynamisme, sa convivialité voire son interactivité, ne parvient à détrôner le papier dans le domaine du plaisir de la lecture. La lecture de l'édition numérique se cantonne à la sphère des professions de l'écrit (qu'elles soient littéraires ou non) parce qu'elles dégagent un profit non négligeable (productivité améliorée, gain de temps, maîtrise plus rapide des corpus) de la valeur ajoutée de ce type de support. Il semble fort peu probable pour le moment que la lecture-plaisir s'oriente vers ce type de support malgré l'omniprésence des ordinateurs dans les foyers, et l'absence de succès des hypertextes fictionnels est là pour le prouver.

54. R. Barthes, op. cit., p. I 2. 
l'outil informatique semblent avant tout servir, voire nourrir, les besoins de l'éditeur scientifique qui possède enfin un outil lui permettant d'atteindre l'érudition, quitte à oublier la présence du lecteur. Pourtant, ce type d'édition doit pouvoir supporter différents modes de lecture : plaisir, curiosité, découverte, critique, experte, génétique... Chacun pouvant être lui-même décliné sur plusieurs niveaux qui sont propres aux documents hypertextuels : lecture de repérage, de balayage, en profondeur's. De même, l'intégration du Web sémantique dans l'édition numérique nécessite certainement une visualisation des relations et des interactions grâce à la représentation logique de l'architecture de l'information. Cette «carte» du document hypertextuel permettra à l'utilisateur de procéder à une lecture schématique ou synthétique de l'ensemble et d'échapper au vertige de la surinformation.

Toutefois, dans le cas de l'inachevé, l'édition numérique joue en faveur du lecteur expert; elle seule peut le dégager de l'emprise éditoriale en faisant de lui «non plus un consommateur, mais un producteur de texte ${ }^{6}{ } » .$. Il ne s'agit pas ici de réaliser le fantasme barthésien du «texte scriptible» (ibid., p. I I), mais plus simplement de donner au lecteur la liberté de composer son propre parcours au sein des brouillons de Lamiel à partir d'un ensemble d'éléments génétiques (paginations multiples, ce qui est écrit, raturé, ajouté, amplifié...), codicologiques (papier, encre, collage, découpage...), historiques et critiques. Cela ne signifie pas que l'éditeur souhaite renier ses responsabilités scientifiques (se protégeant ainsi des remarques du prochain éditeur de Lamiel), puisqu'un texte sera proposé au lecteur si celui-ci souhaite suivre nos propositions; mais elles ne seront qu'un résultat de notre propre hypothèse de lecture du texte, vérifiable, modifiable, à corriger peut-être!

55. M. Cartier, «Trois pôles, trois angles d'analyse, pour saisir l'impact de la culture numérique sur notre société», [en ligne]

56. R. Barthes, op. cit., p. Io. 